\title{
AIDS plagued by journalists
}

This is a shortened version of a letter sent to The Times (London) but not published.

SIR - It was rather bewildering to read the article in The Times of 18 December 1993 by Simon Jenkins, that the main problem with AIDS is that it is "plagued by politics". Jenkins fails to appreciate that AIDS is caused by a viral infection of unparalleled and horrendous consequences to the infected individual in particular and the human race in general.

In the case of other diseases caused by microorganisms that affected large numbers of people before the discovery of antibiotics and the development of protective vaccines, most of the affected died within a relatively short time after infection, whilst those who survived acquired - by and large - a lifelong protective immunity and were not infectious to others. In contrast, infection with the agent that leads to the development of AIDS invariably results in an incubation period of several years, during which time the individuals may be unaware of their infected and infectious state and infect their sexual partners.

Although HIV was first isolated in France 11 years ago, we have learnt an enormous amount about this virus and the way in which it causes a breakdown of the patient's immune system. The recognition of its importance, and corresponding financial support, led to the discovery that HIV infection invariably leads to AIDS. As a result we are also in a position to appreciate why, despite enormous efforts and 9 years of investment in vaccine development, we are no nearer to having either a protective vaccine or an effective drug that could prevent the progression to AIDS, let alone provide a cure.

The application of molecular biology to HIV research has enabled us to recognize that, unlike other infectious agents, the causative agent of AIDS continuously mutates in the body of the infected. This has led to the recognition that each individual HIV has its own molecular fingerprint. It also explains why, for example, AZT has, at best, short-term benefit, because the virus in AZT-treated individuals develops mutants within 6-12 months which become resistant.

It is bewildering that Jenkins can write that HIV infection does not lead to AIDS when we already know that the deadly disease develops in all heterosexual recipients of contaminated blood or blood products who do not belong to the Sunday Times' "lifestyle high-risk group". In the "high-risk group" we already know that more than 90 per cent will develop AIDS within 10 years of infection. Jenkins also fails to appreciate that more than 90 per cent of the men and women around the world who have contracted AIDS do not belong to the Sunday Times' "lifestyle" group because they contracted it during normal heterosexual intercourse. It is, therefore, incomprehensible that Jenkins can write that "no causal chain has been proved" between HIV and AIDS when it had been established some 10 years previously.

It is difficult to understand why Jenkins repeats the Sunday Times accusation that Nature has "led a propaganda war that may have unnecessarily stigmatized millions of HIV-positive people". It is not unreasonable to expect the previous editor of The Times to know that progress in any discipline of science or medicine is based on earlier knowledge, and that any claim to discovery always has to be reproduced by others in the same field before it is accepted. For Jenkins to assume, therefore, that all the physicians and medical scientists involved in AIDS research and treatment are wrong is absurd.

Jenkins argues that we should take note of what Dr Peter Duesberg has to say, as if we were dealing with a philosophical or theological rather than a scientific and medical question on which, as said, all the necessary evidence has been provided to prove some 10 years ago that HIV causes AIDS. Jenkins also repeats the Sunday Times' absurdity that HIV-infected haemophiliacs are dying because "they are bombarded with alien blood", not realizing that haemophiliacs who are HIV-negative have a normal lifespan, while almost all those who become infected with HIV develop AIDS and die early, even children and teenagers who have been "bombarded" for a short period of time.

Another incomprehensible argument by Jenkins against the role of HIV in AIDS is, "why, after 10 years' intensive research, are we no nearer the answer?" We have made considerable progress in understanding the disease, although little progress towards a cure - but the same is true after many more years of research into the common cold, various herpes infections, some of the major cancers and multiple sclerosis.

Jenkins quotes recent statistics from Cambridge about HIV infection but fails to note that, in the previous year, 37 per cent of new HIV infections were in heterosexuals while, early on, the UK infection rate in heterosexuals was in only single figures. The number of AIDS cases in Britain, 5,000, reflects the HIV infections that took place 7-10 years ago, not those occurring now, whose consequences will be seen between 2000 and 2005. Meanwhile, the present number of people with AIDS creates a false sense of security, aided by campaigns such as those of the
Sunday Times, encouraging people to disregard the risks and spread the infection further.

Largely through heterosexual intercourse, the spread of HIV infection and AIDS has already had a devastating effect in some parts of Africa, about which Jenkins remains silent. Heterosexual HIV and AIDS are also increasing at an alarming rate in countries such as India, Brazil and Thailand. The rates of HIV infection are lower in the West but are continuously increasing: in some US cities, AIDS is becoming the major cause of death even in women aged 25-40.

It is about time that The Times and the Sunday Times realized that, through badly informed and irresponsible journalism, they could mislead millions of their readers about HIV infection and AIDS. In the absence of a cure, and without a prospect for an effective vaccine, prevention is the only defence against this formidable disease. Informed, responsible journalists can play an important role in reducing the rate of spread of AIDS.

\section{A. Karpas}

Department of Haematology,

University of Cambridge,

Hills Road, Cambridge CB2 2QH, UK.

SIR - Because of your interest in our reports on HIV and AIDS, I should like to make it clear that, contrary to some reports, the Sunday Times has never presumed to pass judgement on whether or not HIV causes AIDS. We do however believe that a strong scientific challenge has been mounted to the consensus view that HIV is the cause of AIDS, and consider the issue so important as to merit wide discussion and examination. We will continue to report views and findings relevant to this debate.

Many such developments occurred over the past 12 months, and as we have been almost alone among the media in challenging the orthodox view that accepts HIV as the cause of AIDS, we have often found ourselves left with a clear field in writing about them. This has given the impression that we are running a campaign on the issue, but the view here is that all our articles have been of legitimate news interest, given our fundamental position that there are real uncertainties over AIDS causation.

I should like to add that my own doubts about HIV's role have grown stronger over the 20 months since we first set out the "dissident" views, not least because of the unreasoning way mainstream science has reacted to the challenge, at first dismissive and then, when the "problem" refused to go away, openly censorious.

\section{Neville Hodgkinson}

(Science Correspondent)

The Sunday Times,

1 Pennington Street,

London E1 9XW, UK 